\title{
Ultracold two-component Fermi gases with a magnetic field gradient near a Feshbach resonance
}

\author{
Hongwei Xiong ${ }^{1,2,3}$, Shujuan Liu $^{1,2,3}$, Weiping Zhang ${ }^{4}$, Mingsheng Zhan ${ }^{1,2}$ \\ ${ }^{1}$ State Key Laboratory of Magnetic Resonance and Atomic and Molecular Physics, \\ Wuhan Institute of Physics and Mathematics, Chinese Academy of Sciences, Wuhan 430071, P. R. China \\ ${ }^{2}$ Center for Cold Atom Physics, Chinese Academy of Sciences, Wuhan 430071, P. R. China \\ ${ }^{3}$ Graduate School of the Chinese Academy of Sciences, Wuhan 430071, P. R. China and \\ ${ }^{4}$ Department of Physics, East China Normal University
}

\begin{abstract}
We study theoretically the ultracold two-component Fermi gases when a gradient magnetic field is used to tune the scattering length between atoms. For ${ }^{6} \mathrm{Li}$ at the narrow resonance $B_{0}=543.25$ $\mathrm{G}$, it is shown that the gases would be in a coexistence of the regimes of BCS, BEC and unitarity limit with the present experimental technique. In the case of thermal and chemical equilibrium, we investigate the density distribution of the gases and show that a double peak of the density distribution can give us a clear evidence for the coexistence of BCS, BEC and unitarity limit.

PACS numbers: 03.75.Ss, 05.30.Fk, 05.30.Jp, 03.75.Hh
\end{abstract}

With the remarkable development of the cooling technique for two-component Fermi gases and Feshbach resonance, the recent experiments [1, 2, 3] have finally realized molecular Bose-Einstein condensation (BEC) and the condensation of fermionic atom pairs on the side of attractive interaction has also been investigated by several experiments [4, 5, 6, 7, 8, 9, 10, 11]. Together with intensive theoretical investigations 12, 13, 14, 15, 16, 17, $18,19,20,21,22,23,24,25,26,27,28,29,30,31$ such as resonance superfluid [16, 17] and universal behavior for the gases with divergent scattering length [25, 26, 27, 28, 29, 30], these experimental advances provide us a quite unique system which will even contribute largely to our understanding on the mechanism of hightemperature superconductors.

For two-component Fermi gases, when the magnetic field is tuned so that the energy of a quasibound molecular state in a closed channel matches the total energy in an open channel, there is a magnetic-field Feshbach resonance 25, 32] which can tune the scattering length from positive to negative over many orders of magnitude. On the side of repulsive interaction (BEC side), there exists molecule which is a short-range fermionic atom pairs. On the side of attractive interaction (BCS side), there would be a superfluid behavior due to the atomic Cooper pairs at sufficient low temperature. On resonance, the absolute value of the scattering length is much larger than the average distance between atoms and one expects a universal behavior for the system in the unitarity limit. In the present experiments on the BCS-BEC crossover, a uniform magnetic field is used to tune the scattering length through the magnetic-field Feshbach resonance. By tuning the uniform magnetic field near the resonant magnetic field, several experiments have investigated the BCS-BEC crossover such as condensate fraction [4, 5], collective excitation [8, 9], pairing gap [10], and heat capacity [1].

For ultracold two-component Fermi gases confined in an optical trap with axial symmetry along the $z$ axis, in the present work, we will investigate the unique prop- erty of the system when the magnetic field to tune the scattering length has a gradient of $\alpha$. For ${ }^{6} \mathrm{Li}$ at the narrow resonance $B_{0}=543.25 \mathrm{G}$, our researches show that the gases can be in a coexistence of the regimes of BCS, BEC and unitarity limit with appropriate parameters. For the gases in thermal and chemical equilibrium, when the pair size is much smaller than the cloud size of the system, the density distribution of the gases is calculated based on the local density approximation.

For Fermi gases with an equal incoherent mixture of the internal states $|1\rangle$ and $|2\rangle$, near a Feshbach resonant magnetic field $B_{0}$, the scattering length is $a(B)=$ $A a_{0}\left(1-w /\left(B-B_{0}\right)\right)$ with $a_{0}$ being the Bohr radius. For the magnetic field in $x$-direction with gradient $\alpha$ along $z$-direction, we have $\vec{B}=\left(B_{0}+\alpha z\right) \vec{e}_{x}$. In this case, the scattering length becomes $z$-dependent and takes the following form:

$$
a(z)=A a_{0}\left(1-\frac{w}{\alpha z}\right) .
$$

If the magnetic field gradient $\alpha$ is positive, when $z<0$ and $a(z) n^{1 / 3}<1$ ( $n$ is the total density distribution of the ultracold gases), there is repulsive interaction between fermionic atoms which corresponds the BEC side, while when $z>0$ and $|a(z)| n^{1 / 3}<1$ there is attractive interaction between fermionic atoms which corresponds to the BCS side. In the regime determined by $|a(z)| n^{1 / 3}>1$, the ultracold gases are in the unitarity limit where the scattering length can be regarded to be divergent and will not appear in the final result of a physical quantity such as chemical potential. Thus, by using the gradient magnetic field and appropriate parameters, there would be a coexistence of the gases in the form of BCS, BEC and unitarity limit.

For the optical trap with angular frequencies being $\omega_{r}\left(=\omega_{x}=\omega_{y}\right)$ and $\omega_{z}$ in the radial and axial directions, when the magnetic field with gradient $\alpha$ is applied to the ultracold gases, the overall external potential for 
the fermionic atom in the internal state $|i\rangle$ is

$V_{|i\rangle}=\frac{1}{2} m \omega_{r}^{2}\left(x^{2}+y^{2}\right)+\frac{1}{2} m \omega_{z}^{2}\left(z-\Delta z_{|i\rangle}\right)^{2}-\mu_{|i\rangle}^{m a g} B_{0}+\Delta V_{|i\rangle}$

where $i=1,2$. Due to the presence of the magnetic field, for different internal state $|i\rangle$, there is a different shift to the optical trap which is determined by $\Delta z_{|i\rangle}=\alpha \mu_{|i\rangle}^{m a g} / m \omega_{z}^{2}$ with $\mu_{|i\rangle}^{m a g}$ being the magnetic moment of the internal state $|i\rangle$. In the above expression, $\Delta V_{|i\rangle}=-\alpha^{2}\left(\mu_{|i\rangle}^{m a g}\right)^{2} / 2 m \omega_{z}^{2}$. One can also get the overall external potential for a molecule

$$
V_{m o l}=m \omega_{r}^{2}\left(x^{2}+y^{2}\right)+m \omega_{z}^{2} z^{2}-\mu_{m}^{m a g} B,
$$

where $\mu_{m}^{m a g}$ is the magnetic moment of the molecule.

The regime of the gases in the unitarity limit can be roughly determined by $|a(z)| n^{1 / 3}>1$. Thus, the length scale for the regime in the unitarity limit can be estimated as $2 l_{z}^{\text {res }}$ with $l_{z}^{\text {res }}=A w n^{1 / 3} a_{0} / \alpha$. Because there is a different shift for the external potential of the internal state $|1\rangle$ and $|2\rangle$, the difference of the shift should be much smaller than $2 l_{z}^{\text {res }}$ so that one can omit safely the effect of the shift $\Delta z_{|i\rangle}$. After a simple calculation, this condition $\left|\Delta z_{|1\rangle}-\Delta z_{|2\rangle}\right|<<2 l_{z}^{\text {res }}$ means that

$$
A w<<\frac{2\left(l_{z}^{r e s}\right)^{2} m \omega_{z}^{2}}{n^{1 / 3} a_{0}\left|\mu_{|1\rangle}^{\text {mag }}-\mu_{|2\rangle}^{\text {mag }}\right|} .
$$

For the typical parameters that $\omega_{z} / 2 \pi \sim 100 \mathrm{~Hz}, n \sim$ $10^{13} \mathrm{~cm}^{-3}$ and $l_{z}^{\text {res }} \sim 10 \mu \mathrm{m}$, the condition given by Eq. (414) requests that $A w$ is smaller than $10 \mathrm{G}$. This means that very narrow resonance for an element is an appropriate choice to investigate the coexistence of the regimes of BCS, BEC and unitarity limit. A careful investigation shows that the mixture of $|1\rangle \equiv\left|F=1 / 2, m_{F}=1 / 2\right\rangle$ and $|2\rangle \equiv\left|F=1 / 2, m_{F}=-1 / 2\right\rangle$ for ${ }^{6} L i$ at the narrow resonance located at $B_{0}=543.25 \mathrm{G}[33]$ can satisfy our request. For this narrow resonance, $A w$ is estimated as 6 G 33]. Thus, in the present work, we will consider twocomponent Fermi gases of ${ }^{6} \mathrm{Li}$ at the narrow resonance $B_{0}=543.25 \mathrm{G}$.

For two-component Fermi gases of ${ }^{6} \mathrm{Li}$ confined in the optical trap, we will investigate the density distribution of the gases by using the condition that the system is in thermal and chemical equilibrium. Our researches show that with appropriate choice of parameters and element for the system, the coexistence of the gases in the form of BCS, BEC and unitarity limit can be clearly shown through the density distribution. In the present experiments, the gases can be cooled far below the critical temperature of molecular BEC and Fermi temperature of the Fermi gases. Thus, we will consider the system at zero temperature to give a clear presentation. For the molecules on the BEC side, the pair size can be regarded as the scattering length. For the atomic pairs in the unitarity limit, the pair size can be estimated as the average distance between atoms [34]. For the atomic Cooper pairs on the BCS side, one can estimate the pair size based on the BCS theory. We have verified that for the parameters used in the present work, the pair size is much smaller than the cloud size. Thus, one can safely use the well-known local density approximation to calculate the density distribution of the system.

To calculate the density distribution of the system, we firstly investigate the chemical potential of the system. In the regime of molecular BEC $\left(a(z) n^{1 / 3}<1\right)$ with density distribution $n_{m}^{B E C}$ and molecular scattering length $a_{m}=0.6 a$ [35], the chemical potential of the molecular $\mathrm{BEC}$ is given by

$$
\mu_{m}^{B E C}=\frac{2 \pi \hbar^{2} a_{m} n_{m}^{B E C}}{m}+V_{m o l}+\Delta \mu B_{0},
$$

where $\Delta \mu=\mu_{m}^{m a g}-\Sigma_{i} \mu_{|i\rangle}^{m a g}$. The last term $\Delta \mu B_{0}$ in the above equation is due to the energy of the bound state in the closed channel when there is no magnetic field. After a simple calculation, one can get the following expression for the chemical potential

$$
\mu_{m}^{B E C}=\frac{2 \pi \hbar^{2} a_{m} n_{m}^{B E C}}{m}+V_{m o l}^{\prime}+\varepsilon_{m},
$$

where $\varepsilon_{m}=-E_{m}$ with $E_{m}=\hbar^{2} / m a^{2}$ being the binding energy of the molecule. $V_{m o l}^{\prime}$ is given by

$$
\begin{gathered}
V_{m o l}^{\prime}=m \omega_{r}^{2}\left(x^{2}+y^{2}\right)+m \omega_{z}^{2}\left(z-\Delta z_{m o l}\right)^{2} \\
-\Sigma_{i} \mu_{|i\rangle}^{m a g} B_{0}+\Delta V_{m o l}
\end{gathered}
$$

where $\Delta z_{m o l}=\alpha\left(\Sigma_{i} \mu_{|i\rangle}^{m a g}\right) / 2 m \omega_{z}^{2}$ and $\Delta V_{m o l}=$ $-\alpha^{2}\left(\Sigma_{i} \mu_{|i\rangle}^{\text {mag }}\right)^{2} / 4 m \omega_{z}^{2}$.

In this regime, the chemical potential of the Fermi gas in the internal state $|i\rangle$ takes the form

$\mu_{f|i\rangle}^{B E C}=\frac{\hbar^{2}\left(6 \pi^{2}\right)^{2 / 3}}{2 m}\left(n_{f|i\rangle}^{B E C}\right)^{2 / 3}+g_{a a} n_{f|i\rangle}^{B E C}+g_{a m} n_{m}^{B E C}+V_{|i\rangle}$,

where $n_{f|i\rangle}^{B E C}$ is the density distribution of the Fermi gas in the internal state $|i\rangle$. In the above expression $g_{a a}=$ $2 \pi \hbar^{2} a / m_{r}$ with $m_{r}=m / 2$ being the reduced mass. In addition, $g_{a m}=0.9 g_{a a}[36$ which is obtained based on the atom-molecule scattering length $a_{a m}=1.2 a[35]$.

In the regime of the unitarity limit where the absolute value of the scattering length is much larger than the average distance between atoms, as pointed out in [30], we assume that the gases is in the mixture of Fermi gases and dimeric gas in chemical equilibrium. Based on the local density approximation at zero temperature, the chemical potential of the Fermi gas with density distribution $n_{f|i\rangle}^{U L}$ takes the form

$$
\mu_{f|i\rangle}^{U L}=\left(1+\beta_{1}\right) \frac{\hbar^{2}\left(6 \pi^{2}\right)^{2 / 3}}{2 m}\left(n_{f|i\rangle}^{U L}\right)^{2 / 3}+V_{|i\rangle},
$$


where $\beta_{1}$ is firstly measured in [25] and $\beta_{1}=-0.56$ based on a quantum Monte Carlo calculation [27]. Omitting the binding energy of the dimer in the unitarity limit, based on the dimensionality analysis, the chemical potential of the dimeric gas with density distribution $n_{d}^{U L}$ can be assumed as [30]

$$
\mu_{d}^{U L}=\left(1+\beta_{2}\right) \frac{\hbar^{2}\left(6 \pi^{2}\right)^{2 / 3}}{2 \times 2 m}\left(n_{d}^{U L}\right)^{2 / 3}+V_{d} .
$$

For the dimeric gas, $V_{d}$ takes the same form as $V_{m o l}^{\prime}$ for the molecular gas. Based on the condition of the chemical equilibrium $\Sigma_{i} \mu_{f|i\rangle}^{U L}=\mu_{d}^{U L}$, one can get the ratio $n_{d}^{U L} / n_{f|i\rangle}^{U L}=\left[4\left(1+\beta_{1}\right) /\left(1+\beta_{2}\right)\right]^{3 / 2}$ on resonance. From the experimental result $n_{d}^{U L} / n_{f|i\rangle}^{U L} \approx 4$ in [5], $\beta_{2}$ is estimated as -0.3 .

In the regime of $\mathrm{BCS}$, the chemical potential can be approximated as

$$
\mu_{f|i\rangle}^{B C S}=\frac{\hbar^{2}\left(6 \pi^{2}\right)^{2 / 3}}{2 m}\left(n_{f|i\rangle}^{B C S}\right)^{2 / 3}+V_{|i\rangle},
$$

where $n_{f|i\rangle}^{B C S}$ is the density distribution of the fermionic atoms on the BCS side.

In the case of thermal and chemical equilibrium for the system, the minimum of the Gibbs free energy means that there is following important relation for the chemical potential:

$$
\mu_{m}^{B E C}=\Sigma_{i} \mu_{f|i\rangle}^{B E C}=\Sigma_{i} \mu_{f|i\rangle}^{U L}=\mu_{d}^{U L}=\Sigma_{i} \mu_{f|i\rangle}^{B C S} \equiv \mu .
$$

To illustrate clearly the density distribution and evidence for an experiment to show the coexistence of the regime of BCS, BEC and unitarity limit, we use the parameters $\omega_{z} / 2 \pi=120 \mathrm{~Hz}$ and $n_{f|1\rangle}^{U L}=0.2 \times 10^{12} \mathrm{~cm}^{-3}$ at the center $z=0$. In addition, the gradient of the magnetic field is chosen as $\alpha=21 \mathrm{G} / \mathrm{m}$. Based on these parameters, $\left|\Delta z_{|i\rangle}\right| / 2 l_{z}^{r e s}<<1,\left|\Delta V_{|i\rangle}\right| / \mu<<1, \Delta z_{m o l}=0$ and $\Delta V_{\text {mol }} / \mu=0$. Thus, one can safely omit $\Delta z_{|i\rangle}, \Delta V_{|i\rangle}$, $\Delta z_{m o l}$ and $\Delta V_{m o l}$.

From the equilibrium condition for the chemical potential given by Eq. (12), one gets the following expression for the density distribution:

$$
\begin{gathered}
n_{m}^{B E C}=\frac{\left(\mu_{e f f}+\hbar^{2} / m a^{2}-2 V_{e x t}\right) m}{2 \pi \hbar^{2} a_{m}}, \\
n_{f|1\rangle}^{B E C}=n_{f|2\rangle}^{B E C} \approx\left[\frac{2 m\left(\mu_{e f f} / 2-V_{e x t}\right)}{\hbar^{2}\left(6 \pi^{2}\right)^{2 / 3}}\right]^{3 / 2}, \\
n_{f|1\rangle}^{U L}=n_{f|2\rangle}^{U L}=\left[\frac{2 m\left(\mu_{e f f} / 2-V_{e x t}\right)}{\left(1+\beta_{1}\right) \hbar^{2}\left(6 \pi^{2}\right)^{2 / 3}}\right]^{3 / 2}, \\
n_{d}^{U L}=\left[\frac{4 m\left(\mu_{e f f}-2 V_{e x t}\right)}{\left(1+\beta_{1}\right) \hbar^{2}\left(6 \pi^{2}\right)^{2 / 3}}\right]^{3 / 2},
\end{gathered}
$$

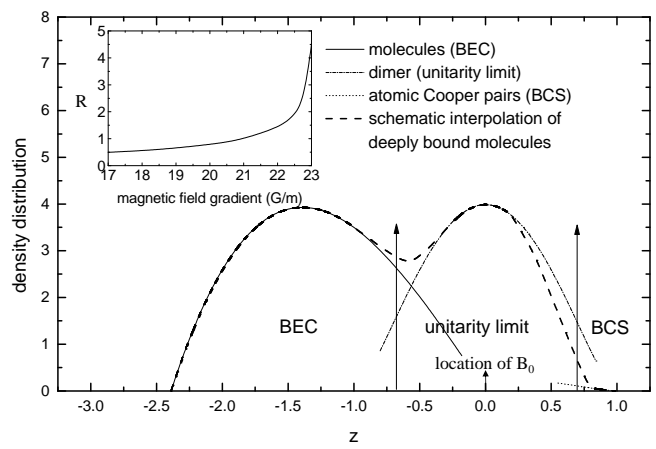

FIG. 1: Shown is the density distribution of the gases when a gradient magnetic field of $21 \mathrm{G} / \mathrm{m}$ is used to tune the atomic scattering length. After the molecules in the regime of BEC, the dimers in the regime of unitarity limit, and the atomic Cooper pairs in the regime of BCS are converted into deeply bound molecules by decreasing non-adiabatically the magnetic field, the thick dashed line illustrates the density distribution of the deeply bound molecules. We see that there is a double peak for the density distribution of the deeply bound molecules which can give us a clear evidence for an experiment to make the system in the coexistence of BEC, BCS and unitarity limit. The ratio of the peak density to valley density is estimated as 1.4. In this figure, the density distribution is in unit of $n_{f|1\rangle}^{U L}(z=0)$, while the coordinate $z$ is in unit of $R_{z}=\sqrt{\mu_{e f f} / m \omega_{z}^{2}}$. In the inset of this figure, shown is the ratio $R$ of the peak density due to molecular BEC and ultracold gases in the unitarity limit for different magnetic field gradient.

$$
n_{f|1\rangle}^{B C S}=n_{f|2\rangle}^{B C S}=\left[\frac{2 m\left(\mu_{e f f} / 2-V_{e x t}\right)}{\hbar^{2}\left(6 \pi^{2}\right)^{2 / 3}}\right]^{3 / 2},
$$

where $V_{\text {ext }}$ takes the form

$$
V_{\text {ext }}=\frac{1}{2} m \omega_{r}^{2}\left(x^{2}+y^{2}\right)+\frac{1}{2} m \omega_{z}^{2} z^{2} .
$$

To give a concise presentation, we have introduced

$$
\mu_{e f f}=2\left(1+\beta_{1}\right) \frac{\hbar^{2}\left(6 \pi^{2}\right)^{2 / 3}}{2 m}\left(n_{f|1\rangle}^{U L}(z=0)\right)^{2 / 3} .
$$

Using the parameters in this paper, shown in Fig.1 is the density distribution of the gases where the density is in unit of $n_{f|1\rangle}^{U L}(z=0)$, while the coordinate $z$ is in unit of $R_{z}=\sqrt{\mu_{e f f} / m \omega_{z}^{2}}$. The arrow shows the location $\pm 0.7 R_{z}$ which is determined by $|a(z)| n^{1 / 3}=1$. The solid line in Fig.1 shows the density distribution of molecular BEC $n_{m}^{B E C}(z<-0.7)$, while the dotdashed line in this figure shows the density distribution $n_{d}^{U L}(-0.7<z<0.7)$ of the dimers in the unitarity limit. The dotted line shows the density distribution of $n_{f|1\rangle}^{B C S}(z>0.7)$. Analogously to the experiments in 
[4, 5], we consider the process that the magnetic field is decreased non-adiabatically so that the fermionic atom pairs (i.e. molecules on the BEC side, dimers in the unitarity limit, and atomic Cooper pairs on the BCS side before the decreasing of the magnetic field) are converted into deeply bound molecules. After this non-adiabatical process, from the density distribution of molecular BEC (solid line) and dimers in the unitarity limit (dot-dashed line), we see that the coexistence of the gases in the form of BCS, BEC and unitarity limit can be clearly shown through the double peaks in the density distribution of the deeply bound molecules. The thick dashed line shows a schematic interpolation of the density distribution of the deeply bound molecules. The evidence for the coexistence of the regimes of BCS, BEC, and unitarity limit can be also shown through the non-symmetric density distribution of the deeply bound molecules about $z=0$ after the non-adiabatic process. The inset in the figure shows the ratio $R$ between the peak density of the regime of $\mathrm{BEC}$ and unitarity limit for different magnetic field gradient. Through the density distribution for different magnetic field gradient, our researches show that for 18 $\mathrm{G} / \mathrm{m}<\alpha<22 \mathrm{G} / \mathrm{m}$, there is obvious double peak density distribution.

In summary, we show that for ${ }^{6} \mathrm{Li}$ at the narrow resonance $B_{0}=543.25 \mathrm{G}$, by using a gradient magnetic field to change the scattering length, one can make the gases become the coexistence of the regimes of BCS, BEC and unitarity limit. In the case of thermal and chemical equilibrium, it is shown that with appropriate parameters there is a double peak in the density distribution of the deeply bound molecules after the non-adiabatic decreasing of the magnetic field. This can give us a clear evidence for the coexistence of the regimes of BCS, BEC and unitarity limit in an experiment.

We acknowledge the useful discussions with Dr. Cheng Chin. This work is supported by NSFC under Grant Nos. 10205011, 10474117 and 10474119, NBRPC under Grant Nos. 2005CB724508 and 2001CB309309.
[1] S. Jochim et al., Science 302, 2101 (2003).

[2] M. Greiner, C. A. Regal, and D. S. Jin, Nature 426, 537 (2003).

[3] M. W. Zwierlein et al., Phys. Rev. Lett. 91, 250401 (2003).

[4] C. A. Regal, M. Greiner, and D. S. Jin, Phys. Rev. Lett. 92, 040403 (2004).

[5] M. W. Zwierlein et al., Phys. Rev. Lett. 92, 120403 (2004).

[6] M. Bartenstein et al., Phys. Rev. Lett. 92, 120401 (2004).

[7] T. Bourdel et al., Phys. Rev. Lett. 93, 050401 (2004).

[8] J. Kinast, S. L. Hemmer, M. E. Gehm, A. Turlapov, and J. E. Thomas, Phys. Rev. Lett. 92, 150402 (2004).

[9] M. Bartenstein et al., Phys. Rev. Lett. 92, 203201 (2004).

[10] C. Chin et al., Science 305, 1128 (2004).

[11] J. Kinast et al., Science 307, 1296 (2005).

[12] A. J. Leggett, J. Phys. C. (Paris) 41, 7 (1980).

[13] P. Nozières and S. Schmitt-Rink, J. Low Temp. Phys. 59, 195 (1985).

[14] H. T. C. Stoof, M. Houbiers, C. A. Sackett, and R. G. Hulet, Phys. Rev. Lett. 76, 10 (1996).

[15] Y. Ohashi and A. Griffin, Phys. Rev. A 67, 033603 (2003); Phys. Rev. A 67, 063612 (2003); Phys. Rev. Lett. 89, 130402 (2002).

[16] E. Timmermans, K. Furuya, P. W. Milonni, and A. K. Kerman, Phys. Lett. A 285, 228 (2001).

[17] M. Holland, S. J. J. M. F. Kokkelmans, M. L. Chiofalo, and R. Walser, Phys. Rev. Lett. 87, 120406 (2001); J. N. Milstein, S. J. J. M. F. Kokkelmans and M. J. Holland, Phys. Rev. A 66, 043604 (2002).

[18] J. Stajic et al., Phys. Rev. A 69, 063610 (2004).

[19] X. Yang, and Y. Wu, Commun. Theor. Phys. 39, 543
(2003).

[20] L. D. Carr, G. V. Shlyapnikov, and Y. Castin, Phys. Rev. Lett. 92, 150404 (2004).

[21] G. M. Bruun, Phys. Rev. A 70, 053602 (2004).

[22] G. M. Falco and H. T. C. Stoof, Phys. Rev. Lett. 92, 130401 (2004).

[23] A. Perali, P. Pieri, and G.C. Strinati, Phys. Rev. Lett. 93, 100404 (2004).

[24] H. Xiong et al., preprint cond-mat/0404565 (2004).

[25] K. M. O'Hara et al., Science 298, 2179 (2002).

[26] H. Heiselberg, Phys. Rev. A 63, 043606 (2001).

[27] J. Carlson, S. Y. Chang, V. R. Pandharipande, and K. E. Schmidt, Phys. Rev. Lett. 91, 050401 (2003).

[28] T. L. Ho and E. J. Mueller, Phys. Rev. Lett. 92, 160404 (2004).

[29] T. L. Ho, Phys. Rev. Lett. 92, 090402 (2004).

[30] H. Xiong, S. Liu and M. Zhan, preprint cond-mat/0411626 (2004).

[31] J. Kinnunen, M. Rodriguez, P. Törmä, Science 305, 1131 (2004).

[32] W. C. Stwalley, Phys. Rev. Lett. 37, 1628 (1976); E. Tiesinga, B. J. Verhaar, and H. T. C. Stoof, Phys. Rev. A 47, 4114 (1993).

[33] K. E. Strecker, G. B. Partridge, and R. G. Hulet, Phys. Rev. Lett. 91, 080406 (2003).

[34] T. L. Ho, Science 305, 1114 (2004).

[35] D. S. Petrov, C. Salomon, and G. V. Shlyapnikov, Phys. Rev. Lett. 93, 090404 (2004).

[36] L. D. Carr, R. Chiaramonte, and M. J. Holland, Phys. Rev. A 70, 043609 (2004). 